OPEN ACCESS

Edited by:

Eytan Wine,

University of Alberta, Canada

Reviewed by:

Firas Rinawi,

Technion Israel Institute of

Technology, Israel

Maria Oana Sasaran

George Emil Palade University of

Medicine, Pharmacy, Sciences and

Technology of Târgu Mureş, Romania

*Correspondence:

Yuhuan Wang

wangyuhuan08@163.com

Ying Huang

yhuang815@163.com

Specialty section:

This article was submitted to

Pediatric Gastroenterology,

Hepatology and Nutrition,

a section of the journal

Frontiers in Pediatrics

Received: 20 July 2021 Accepted: 02 November 2021

Published: 13 December 2021

Citation:

Hu W, Feng Y, Ye Z, Tang Z, Qian L,

Wang $Y$ and Huang $Y$ (2021) The

Association Between Genetic

Variants, Pharmacokinetics, and Infliximab Efficacy in Pediatric Patients

With Crohn's Disease in China.

Front. Pediatr. 9:744599.

doi: 10.3389/fped.2021.744599

\section{The Association Between Genetic Variants, Pharmacokinetics, and Infliximab Efficacy in Pediatric Patients With Crohn's Disease in China}

\author{
Wenhui Hu, Yan Feng, Ziqing Ye, Zifei Tang, Lai Qian, Yuhuan Wang* and Ying Huang* \\ Department of Gastroenterology, Pediatric Inflammatory Bowel Disease Research Center, National Children's Medical Center, \\ Children's Hospital of Fudan University, Shanghai, China
}

Background: Infliximab is an effective therapy for Crohn's disease (CD). Early noninvasive predictors of disease remission allow for modification of treatments. The aim of this study was to investigate the associations between genetic variants, pharmacokinetics, and infliximab efficacy in pediatric patients with $C D$.

Methods: This retrospective observational study included CD patients under infliximab therapy between August 2015 and December 2020. Information on demographics, laboratory tests, medication data, and disease activity index was collected. The trough levels of infliximab (TLI) and antibodies to infliximab (ATI) were measured at week 14, and reactive drug monitoring was performed during follow-up. Ten singlenucleotide polymorphisms involved in the NF-kB-mediated inflammatory response, pharmacokinetics, and therapeutic response to infliximab were genotyped.

Results: A total of 62 pediatric CD patients were enrolled. The clinical remission (CR) rate was 69.4 and $63.2 \%$ at week 14 and week 30, respectively. TLI at week 14 was significantly independently associated with $\mathrm{CR}$ at week 14 and mucosal healing $(\mathrm{MH})$ at week 30 ( $p=0.007$ and $p=0.025$, respectively). The optimal TLI threshold level capable of distinguishing between the CR and non-CR groups was $2.62 \mu \mathrm{g} / \mathrm{ml}(p<0.001$, area under the curve $=0.79$, sensitivity $=69.2 \%$, specificity $=78.9 \%$, while that capable of distinguishing between the $\mathrm{MH}$ and non-MH groups was $3.34 \mu \mathrm{g} / \mathrm{ml}(p<0.001$, area under the curve $=0.85$, sensitivity $=78.6 \%$, specificity $=79.4 \%$ ). Rs3397 in TNFRSF1B was associated with time to ATI production in CD patients $(p<0.001)$.

Conclusions: Higher TLI contributed to achieving MH. Genotyping rs3397 in TNFRSF1B may identify patients who are prone to generating immunogenicity to drugs.

Keywords: Crohn's disease, infliximab, children, single nucleotide polymorphisms, pharmacokinetics 


\section{INTRODUCTION}

Crohn's disease (CD) is a chronic inflammatory disease characterized by relapsing and remitting inflammation of the gastrointestinal tract (1). Infliximab (IFX), a chimeric humanmurine monoclonal antibody against tumor necrosis factor alpha $(\mathrm{TNF}-\alpha)$, has been used to induce and maintain remission in patients with CD (2). Administration of IFX rapidly improves clinical symptoms and has a durable effect for up to 54 weeks with an acceptable safety profile (3). However, a systematic review demonstrated that the annual risk of loss of response was estimated to be $13 \%$ per patient-year (4). Since IFX is the most commonly used biological agent for pediatric $\mathrm{CD}$ in China, optimization of IFX therapy seems to be mandatory.

Early non-invasive predictors of clinical and endoscopic remission can allow timely IFX treatment modifications in pediatric CD (5). Therapeutic drug monitoring (TDM), which is based on measurement of trough levels of IFX (TLI) and antibodies to IFX (ATI), is emerging as an important tool to optimize the efficacy of IFX in pediatric CD $(6,7)$. A prospective study suggested that measuring ATI and TLI might predict clinical and endoscopic remission in CD (8). Clinical interventions based on reactive TDM resulted in improved rates of disease remission (9). Higher titers of anti-drug antibodies are associated with lower drug concentrations, which may lead to immune-mediated pharmacokinetic failure (10).

Several recent studies identified genetic variants associated with abnormal therapeutic TLI and increased susceptibility to immunogenicity. The HLA-DQA1*05 allele was identified among European patients with luminal $\mathrm{CD}$ as being associated with an increased risk of developing antibodies against antiTNF agents (11). The single-nucleotide polymorphisms (SNPs) rs5030728 (TLR4) and rs11465996 (LY96) were found to be associated with subtherapeutic TLI in pediatric CD (12). Moreover, pharmacogenetics was investigated to predict the response before anti-TNF treatment initiation. Polymorphisms in TNFRSF1B, IL10,IL17, and IL6 were found to be associated with long-term response to anti-TNF therapy among pediatric CD patients, while this correlation was not found in adults, which revealed that the pharmacogenetics differs between adults and children (13). Overall, more studies are needed regarding the potential contribution of pharmacogenetics to IFX treatment for pediatric CD.

The aim of this retrospective study was to investigate the association between genetic variants, pharmacokinetics, and clinical outcomes during IFX treatment. We developed a nomogram for predicting $\mathrm{MH}$ at week 30 and explored the effect of SNP within a list of 10 genes on pharmacokinetics to IFX treatment in pediatric patients with $\mathrm{CD}$ in China.

\section{METHODS}

\section{Patients}

This study was a retrospective single-center study conducted at the Children's Hospital of Fudan University, National Children's Medical Center, Shanghai, China between August 1, 2015, and December 1, 2020. The inclusion criteria were as follows: (1) age
$<18$ years, (2) clinical diagnosis of $\mathrm{CD}$ according to the Porto criteria and Paris classification $(14,15),(3)$ at least 14 weeks of IFX treatment, (4) biologic-naïve patients, and (5) written informed consent. Exclusion criteria were the presence of definite germline mutation and any infections preventing the use of IFX.

This study was approved by the ethical committee at the Children's Hospital of Fudan University. Informed consent for participation and sample collection was obtained from their parents.

\section{Therapeutic Protocol}

An induction phase with administration of IFX $5 \mathrm{mg} / \mathrm{kg}$ at weeks 0,2 , and 6 was followed by a maintenance phase, when IFX $5 \mathrm{mg} / \mathrm{kg}$ was intravenously administered every 8 weeks. From August 2015 to January 2017, IFX was used for inducing and maintaining remission in moderate-to-severe pediatric $\mathrm{CD}$ and in patients with a high risk of poor outcomes, including extensive disease, deep colonic ulcers, structuring, and penetrating lesions.

Increasing evidence supports that exclusive enteral nutrition (EEN) has statistically equivalent efficacy to the use of corticosteroids in inducing remission (16). EEN demonstrated better efficacy in pediatric CD patients compared to adults, as well as the added benefit of providing nutritional support during the growth phase $(16,17)$. Therefore, first-line EEN has been used for induction therapy in active luminal CD patients at our center since February 2017 (Supplementary Figure S1). IFX is subsequently considered for patients who (1) cannot tolerate EEN, (2) fail to achieve remission after EEN induction therapy, and (3) present high-risk factors, even in remission after induction of EEN.

Considering the potential risks of lymphoma and tuberculosis in combination with thiopurine (18), anti-TNF monotherapy is prioritized at our center. In response to the clinical evidence of disease recurrence with abnormal TLI and ATI, concomitant immunosuppressive therapy (azathioprine and methotrexate) is permitted, the IFX dose can be intensified (up to $10 \mathrm{mg} / \mathrm{kg}$ per dose), and the interval between infusions can be shortened.

\section{Disease Activity Assessments and Outcome Measures}

Laboratory markers were monitored at each infusion, including C-reactive protein (CRP) levels, erythrocyte sedimentation rate (ESR), hematological examination, and serum biochemical indices. Clinical evaluation was performed before induction treatment and at weeks 14,30, and 54 of IFX therapy via weighted Pediatric Crohn's Disease Activity Index (wPCDAI) with values indicating clinical remission $(<12.5$ points, CR), mild (12.5-40 points), moderate ( $>40-57.5$ points), and severe disease ( $>57.5$ points) (19). Colonoscopy was performed before EEN/corticosteroid induction therapy at weeks 0 and 30 of IFX therapy. A time window of endoscopic reexamination between weeks 30 and 38 was permitted. Mucosal healing $(\mathrm{MH})$ was defined as the Simple Endoscopic Score for Crohn Disease (SESCD) $<3$ points $(20,21)$.

The primary outcome was $\mathrm{MH}$ at week 30 of IFX therapy. Secondary outcomes included CR at weeks 14, 30, and 54 of IFX therapy and the time of ATI development. 


\section{Measurement of TLI and ATI}

TLI and ATI examinations were performed prior to infusion at week 14, and reactive drug monitoring was performed during follow-up. Peripheral blood samples were collected before IFX infusions and stored at $4^{\circ} \mathrm{C}$. TLI and ATI were detected using the commercially available immunochromatographic assay, and serum TNF- $\alpha$ was measured using an enzyme-linked immunosorbent assay (HeRui IBD, Suzhou, China). The normal ranges for TLI, ATI, and TNF- $\alpha$ were $>1.0 \mu \mathrm{g} / \mathrm{ml},<30 \mathrm{ng} / \mathrm{ml}$, and $<8.1 \mathrm{pg} / \mathrm{ml}$, respectively.

\section{DNA Isolation and SNP Genotyping}

DNA was extracted from $250 \mu \mathrm{l}$ of patient peripheral blood using a blood purification kit (Omega, D3392-01) according to the manufacturer's instructions. Polymorphisms were genotyped on the Agena MassARRAY ${ }^{\circledR}$ platform, conducted by OE Biotech Co., Ltd (Shanghai, China). Briefly, the initial multiple polymerase chain reaction (PCR) amplification was conducted using an Agena amplification kit. Subsequently, shrimp alkaline phosphatase treatment was performed to remove free deoxyribonucleoside triphosphates in the reaction system. Then, the single-base extension reaction was conducted, and resin purification was performed. Finally, the PCR product was genotyped by MALDI-TOF mass spectrometry and analyzed using Agena Typer 4.0 software.

A total of 10 SNPs were selected from the previous literature, namely, TNFRSF1A (rs4149570), TNFRSF1B (rs3397 and rs1061624), TLR4 (rs5030728), TLR2 (rs3804099), IL6 (rs10499563), IL17A (rs2275913), IL10 (rs1800872 and rs3024505), and HLADQA1 (rs2097432), which are involved in the NF- $\kappa$ B-mediated inflammatory response and are associated with pharmacokinetics and the therapeutic response to IFX $(11,13,22-24)$.

\section{Statistical Analysis}

Statistical analysis was performed using SPSS 20.0 for Windows (IBM, Somers, NY). Continuous clinical and demographic variables are expressed as the median and standard deviation or as the median and interquartile range (IQR). The categorical variables are expressed as percentages. The Mann-Whitney test was applied for the comparison of two groups. Multivariate logistic regression analysis was performed on all variables with $p<0.05$ in the univariate analysis. Receiver operating characteristic (ROC) curve analysis was performed to determine the best threshold of TLI for identifying CR or MH, as well as to calculate the specificity, sensitivity, likelihood ratio, and area under the ROC curve (AUROC). Spearman correlation analysis was performed to observe the correlation between TLI and ATI. A nomogram was formulated according to the results of the multivariate logistic regression model by applying the $\mathrm{R}$ package rms (25). The performance of the nomogram was internally validated in terms of calibration and discrimination capacity. The bootstrap resampling strategy was applied for the calibration plot and estimation of AUROC with 2,000 repetitions. For survival analysis, the Kaplan-Meier method was applied to estimate the survival function, and the log-rank test was used for comparison. A two-sided $p<0.05$ significance level was used throughout.
TABLE 1 | Patient's characteristics.

\begin{tabular}{|c|c|}
\hline Characteristic & $N(\%)$ \\
\hline Gender (male/female) & $39 / 23$ \\
\hline Age of disease onset (years), median (IQR) & $11.00(8.00-12.41)$ \\
\hline Age at infliximab initiation (years), median (IQR) & $12.24(10.00-14.35)$ \\
\hline Duration of IFX therapy (months), median (IQR) & $20.07(12.60-29.87)$ \\
\hline wPCDAI before induction therapy & $37.18 \pm 18.79$ \\
\hline SES-CD before induction therapy & $13.00(7.00-19.00)$ \\
\hline \multicolumn{2}{|l|}{ Laboratory markers before IFX therapy } \\
\hline White blood cell $\left(\times 10^{9} / \mathrm{l}\right)$ & $7.05(5.80-10.50)$ \\
\hline Hemoglobin (g/l) & $117.89 \pm 16.30$ \\
\hline Albumin $(g / l)$ & $39.19 \pm 4.43$ \\
\hline C-reactive protein (mg/l) & $10.50(8.0-24.5)$ \\
\hline Erythrocyte sedimentation rate (mm/h) & $41.50(18.75-73.25)$ \\
\hline \multicolumn{2}{|l|}{ Location according to the paris classification } \\
\hline L1 & 8 (12.9\%) \\
\hline L2 & $19(30.6 \%)$ \\
\hline L3 & $34(54.8 \%)$ \\
\hline $\mathrm{L} 4 \mathrm{a}$ & $29(46.8 \%)$ \\
\hline \multicolumn{2}{|l|}{ Behavior according to the paris classification } \\
\hline B1 & $53(85.5 \%)$ \\
\hline B2 & $6(9.7 \%)$ \\
\hline B3 & $3(4.8 \%)$ \\
\hline B2B3 & $0(0.0 \%)$ \\
\hline$P$ & $14(22.6 \%)$ \\
\hline \multicolumn{2}{|l|}{ Concomitant therapy } \\
\hline 5-Aminosalicylic acid & 58 (93.6\%) \\
\hline Azathioprine & 8 (12.9\%) \\
\hline Methotrexate & $1(1.6 \%)$ \\
\hline None & $4(6.5 \%)$ \\
\hline
\end{tabular}

IQR, interquartile range; wPCDAI, weighted Pediatric Crohn's Disease Activity Index; SES-CD, Simple Endoscopic Score for Crohn Disease.

\section{RESULTS}

\section{Clinical Characteristics and Outcomes}

Among the 80 patients treated with IFX at our center, $62 \mathrm{CD}$ patients met the eligibility criteria. Blood samples were obtained from 58 patients. The median duration of IFX treatment was 20.1 months (IQR: 12.6-29.87). The demographic and clinical characteristics of the enrolled patients are shown in Table 1. During the first year of IFX treatment, 9 patients (14.5\%) received concomitant immunosuppressive treatment and 27 patients (43.5\%) received IFX dose intensification or interval shortening.

Among the 18 (29.0\%) patients receiving EEN and 3 (4.8\%) patients receiving corticosteroids as induction therapy, there was a significant decrease in wPCDAI and SES-CD after induction therapy (wPCDAI: 40.0, IQR: $26.25-53.75$ vs. 7.5, IQR: $0.00-$ 23.75, $p<0.001$; SES-CD: 19.0, IQR: 13.0-20.0 vs. 5.5, IQR: $3.0-$ $14.75, p=0.002)$. A total of $12(57.1 \%)$ patients were in CR before subsequent IFX therapy, and $17(81.0 \%)$ patients achieved CR at week 14 of IFX therapy. In 41 (66.1\%) patients who received antiTNF therapy for inducing remission, there was also a significant decrease in measured values of wPCDAI, platelets, CRP, and ESR 
$(p<0.001)$ after induction therapy, whereas $26(63.4 \%)$ patients were in CR after IFX induction therapy. In total, $69.4 \%$ of patients were in CR at week 14 of IFX treatment. Among the 49 patients who underwent endoscopic reexamination at week $30, \mathrm{MH}$ was observed in $30.6 \%$ of patients. At week $54,66.7 \%$ of patients (32/48) achieved CR.

\section{Factors Associated With Disease Remission}

In order to identify risk factors that were associated with both short-term and long-term remission, we applied a two-step strategy based on a logistic regression model. In univariate logistic regression analysis, the white blood cell count, erythrocyte sedimentation rate, and wPCDAI before IFX initiation were significantly associated with $\mathrm{CR}$ at week 14 (Table 2). TLI was associated with CR at week 14 and MH at week 30 (both $p=0.002$ ). However, the association was lost between TLI and CR at week $54(p=0.188)$. EEN/corticosteroid induction therapies were not statistically significant in CR and $\mathrm{MH}$. Variables with a $p<0.05$ in each univariate regression analysis were included in the multivariate analysis, as presented in Tables 2, 3.

Multivariate logistic regression analysis was then performed to assess the independent factors associated with clinical response and MH. Higher TLI at week 14 independently predicted a higher probability of $\mathrm{MH}$ at week 30 (Table 3). Consistent with the findings from univariate analyses, TLI was confirmed to be an independent predictor for short-term treatment response (Table 2). The median TLI at week 14 was significantly higher in patients who achieved CR at week 14 compared with those who did not (3.50 vs. $0.47 \mu \mathrm{g} / \mathrm{ml}, p<0.001)$, as well as in patients with $\mathrm{MH}$ compared with those without $\mathrm{MH}(4.35 \mathrm{vs} .1 .43 \mu \mathrm{g} / \mathrm{ml}$, $p<0.001)$.

ROC curves were constructed to determine the optimal cutoff value of TLI at week 14 for predicting $\mathrm{CR}$ and $\mathrm{MH}$ in pediatric CD patients treated with IFX. The optimal TLI threshold level capable of distinguishing between the CR and non-CR groups at week 14 was $2.62 \mu \mathrm{g} / \mathrm{ml}$ with an AUROC of $0.79(p<$ 0.001 , sensitivity $=69.2 \%$, specificity $=78.9 \%$ ). Furthermore, the threshold value of TLI at week 14 capable of differentiating patients with or without $\mathrm{MH}$ at week 30 was $3.34 \mu \mathrm{g} / \mathrm{ml}$ with an AUROC of $0.85(p<0.001$, sensitivity $=78.6 \%$, specificity $=$ $79.4 \%$ ) (Figure 1).

No significant differences in $\mathrm{CR}$ or $\mathrm{MH}$ rate in the first year were found on the basis of SNP genotype.

\section{Prognostic Nomogram for MH at Week 30}

A nomogram was then constructed to facilitate the prediction of $\mathrm{MH}$ at week 30. The variable selection was in accordance with the results of the unadjusted logistic regression model of $\mathrm{MH}$ at week 30. Factors including ESR, albumin, and SES-CD before induction treatment, as well as wPCDAI and TLI at week 14, were used as dichotomous variables with the cut-off values determined by Youden index. The final nomogram is depicted in Figure 2A. The calibration capacity of the multivariate model was visually inspected using the calibration chart (Figure 2B) showing great agreement between actual and predicted probabilities. On the

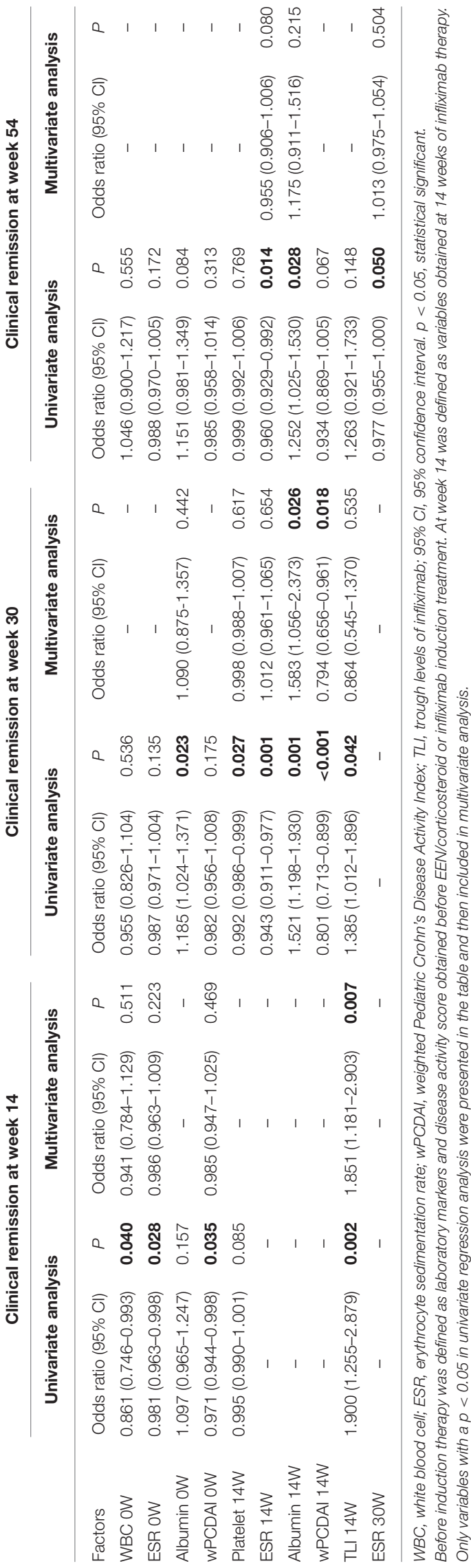


TABLE 3 | Factors associated with mucosal healing at week 30.

\begin{tabular}{|c|c|c|c|c|}
\hline \multirow[b]{2}{*}{ Factors } & \multicolumn{2}{|l|}{ Univariate analysis } & \multicolumn{2}{|l|}{ Multivariate analysis } \\
\hline & Odds ratio (95\% confidence interval) & $\boldsymbol{P}$ & Odds ratio (95\% confidence interval) & $\boldsymbol{P}$ \\
\hline Albumin & $1.157(1.010-1.326)$ & 0.035 & $1.230(0.858-1.763)$ & 0.260 \\
\hline ESR & $0.971(0.947-0.995)$ & 0.018 & $0.987(0.943-1.033)$ & 0.565 \\
\hline ESR & $0.917(0.865-0.973)$ & 0.004 & $1.011(0.910-1.122)$ & 0.845 \\
\hline Albumin & $1.310(1.032-1.663)$ & 0.026 & $1.166(0.708-1.923)$ & 0.546 \\
\hline wPCDAl & $0.873(0.785-0.972)$ & 0.014 & $0.867(0.668-1.125)$ & 0.281 \\
\hline TLI & $2.333(1.355-4.016)$ & 0.002 & $2.759(1.136-6.703)$ & 0.025 \\
\hline
\end{tabular}

ESR, erythrocyte sedimentation rate; SES-CD, Simple Endoscopic Score for Crohn Disease; WPCDAl, Weighted Pediatric Crohn's Disease Activity Index; TLI, trough levels of infliximab. $p<0.05$, statistical significant.

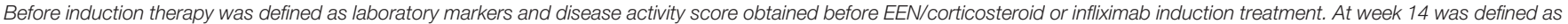
variables obtained at 14 weeks of infliximab therapy. Only variables with a $p<0.05$ in univariate regression analysis were presented in the table and then included in multivariate analysis.

\section{A ROC Curve}

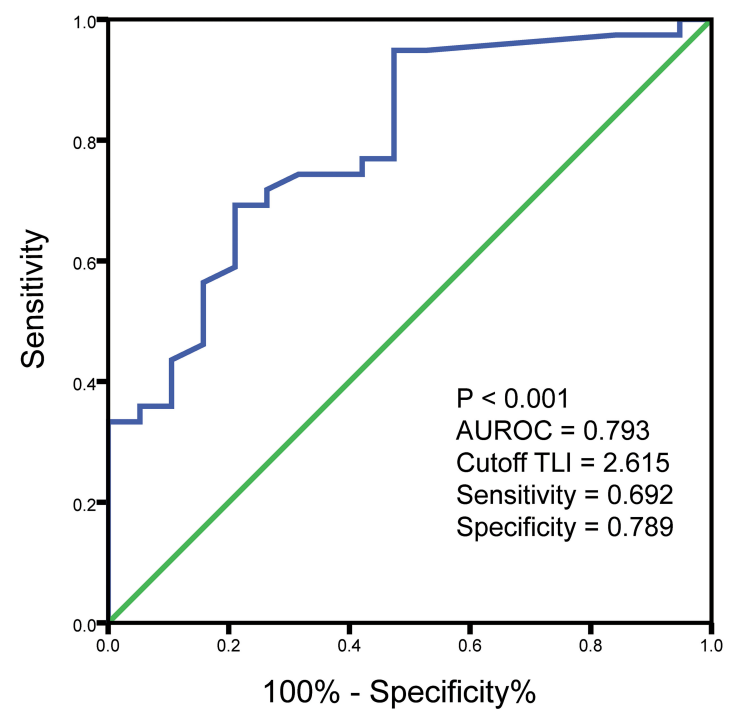

B

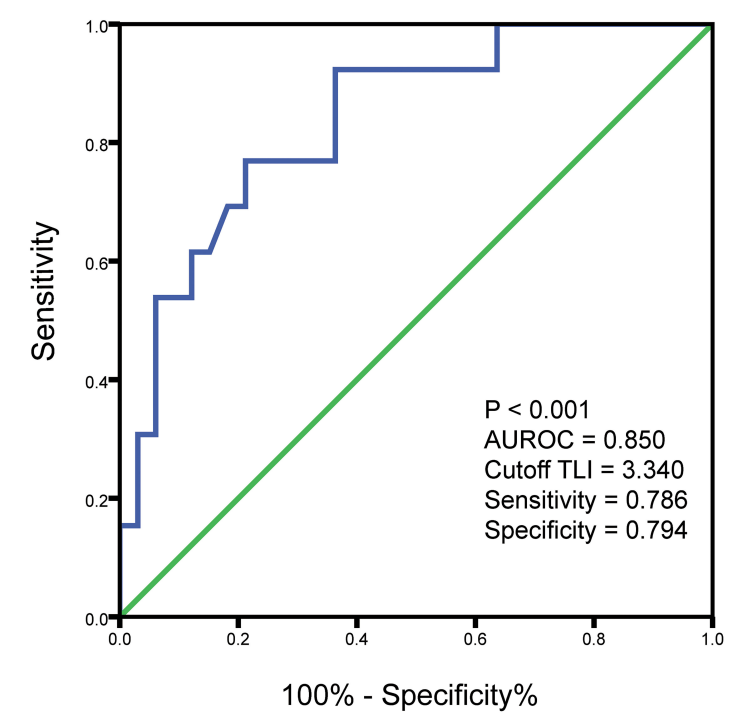

FIGURE 1 | ROC curves showing the association of TLI with clinical outcomes. (A) ROC curve to identify the threshold value of TLI associated with clinical remission. (B) ROC curve to identify the optimal cutoff for TLI associated with endoscopic remission. ROC, receiver operating characteristic; TLI, trough levels of infliximab; AUROC, area under the ROC curve.

other hand, the discrimination capacity of the model was assessed by ROC analysis with an AUROC reaching 0.93 (95\% confidence interval: 0.84-0.98) (Figure 2C). Of note, we observed that the predicted probability closely approximated the actual observation in the lower range of probability from 0 to 0.4 , indicating that our model may potentially help identify patients failing to achieve $\mathrm{MH}$ at the earliest time point of week 14 .

\section{SNPs Associated With Pharmacokinetics to IFX in CD}

The ATI level was below the detection limit $(<4 \mathrm{ng} / \mathrm{ml})$ in $74.2 \%(46 / 62)$ of patients at week 14. Although ATI at week
14 was not associated with disease remission at weeks 14, 30, and 54, ATI levels were inversely correlated with TLI during the maintenance treatment (Pearson test $p=0.002, \rho=-0.392$ ). As the IFX treatment progressed, 16 of 62 patients (25.8\%) showed positively increased antibody levels (defined as ATI $>30 \mathrm{ng} / \mathrm{ml}$ ). The median time from inception to ATI detection in our population was 13.48 months (IQR: 8.57-23.02).

In the time-to-event analysis, one SNP (rs3397) in TNFRSF1B was significantly associated with the time of ATI production. However, associations between the nine other SNPs and ATI development were not statistically significant. TNFRSF1B CC genotype (rs3397) was predictive of significantly earlier ATI 
A

\section{Points}

$\mathrm{ESR}^{1}$

$\mathrm{ALB}^{1}$

SES-CD ${ }^{1}$

WPCDAI ${ }^{2}$

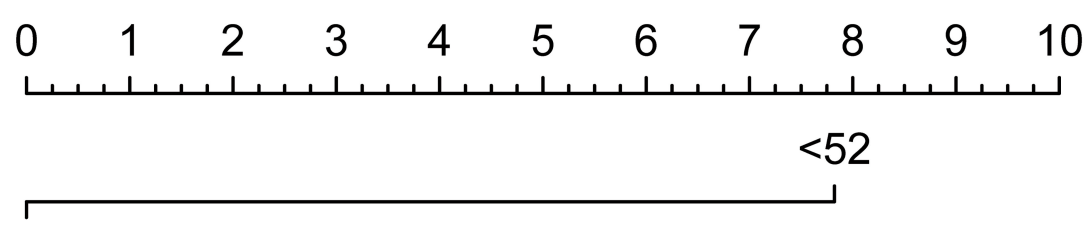

higher

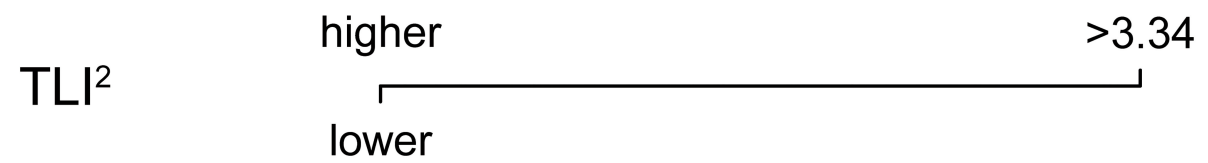

Total Points

lower

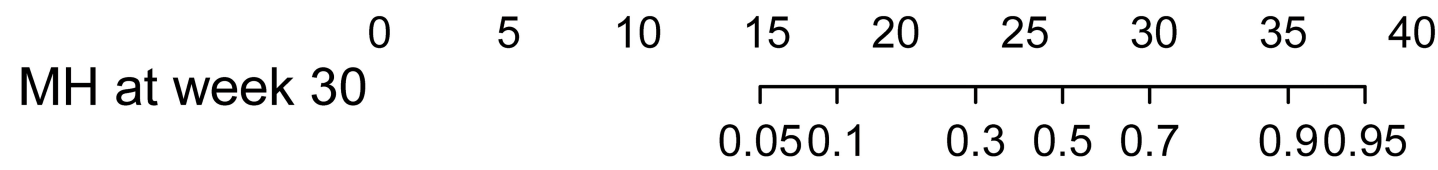

B

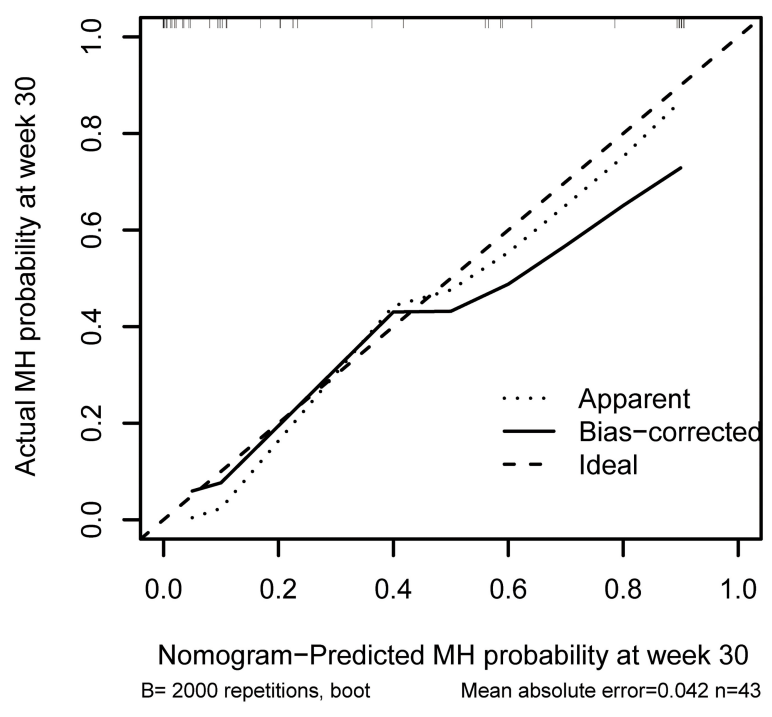

C

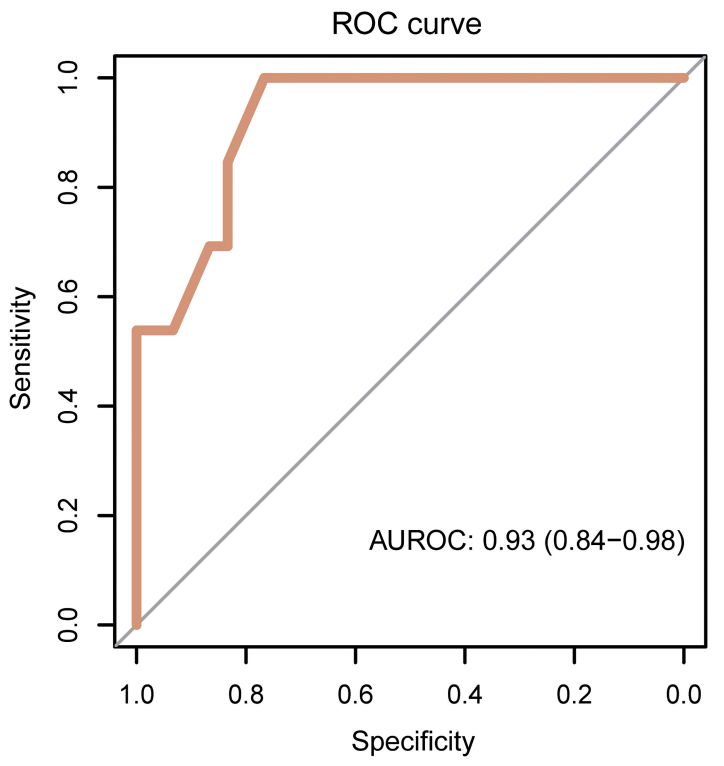

FIGURE 2 | Development, calibration, and performance of nomogram. (A) Week 30 MH nomogram. Each variable is assigned a score on the "points" axis. The sum point corresponds to the estimated probability of $\mathrm{MH}$ at week 30. ESR, ALB, and SES-CD were evaluated before induction treatment. wPCDAI and TLI were determined at week 14. (B) Calibration plot for internal validation of the nomogram. Curves represent observed vs. predicted probabilities. (C) ROC curve indicating the discrimination capacity of the nomogram. AUC and its 95\% confidence interval were estimated by bootstrap resampling with 2,000 repetitions. $\mathrm{MH}$, mucosal healing; ESR, erythrocyte sedimentation rate; ALB, albumin; SES-CD, Simple Endoscopic Score for Crohn Disease; wPCDAI, weighted Pediatric Crohn's Disease Activity Index; AUC, area under the curve.

occurrence compared to the TC or TT genotype with the $p<$ 0.001 (Figure 3A). We noticed that the probability of remaining antibody-free 3 years after the start of IFX therapy was more than $50 \%$ in patients with a TC or TT genotype. After adjusting for variables including hemoglobin, erythrocyte sedimentation rate before IFX treatment, TLI at week 14, serum TNF- $\alpha$ level at 
A

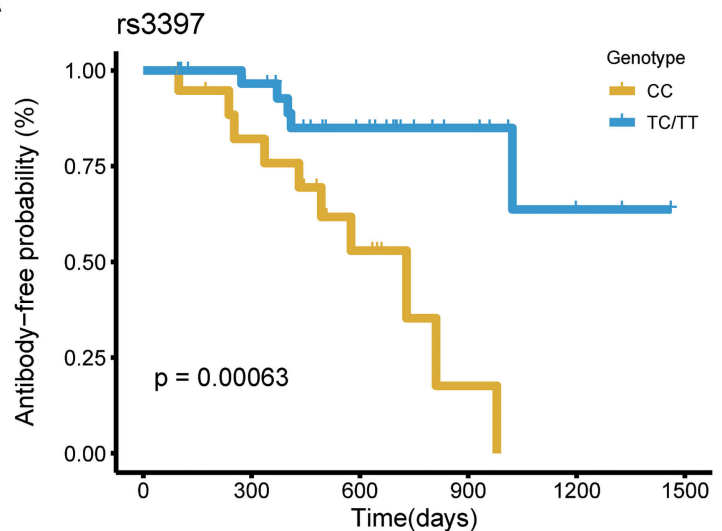

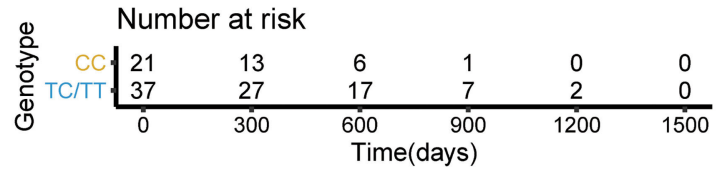

B

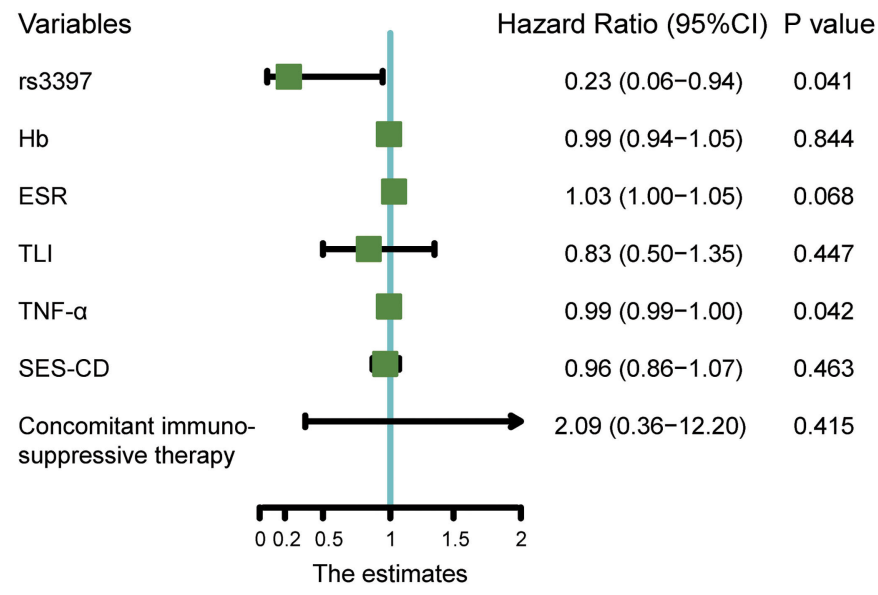

C

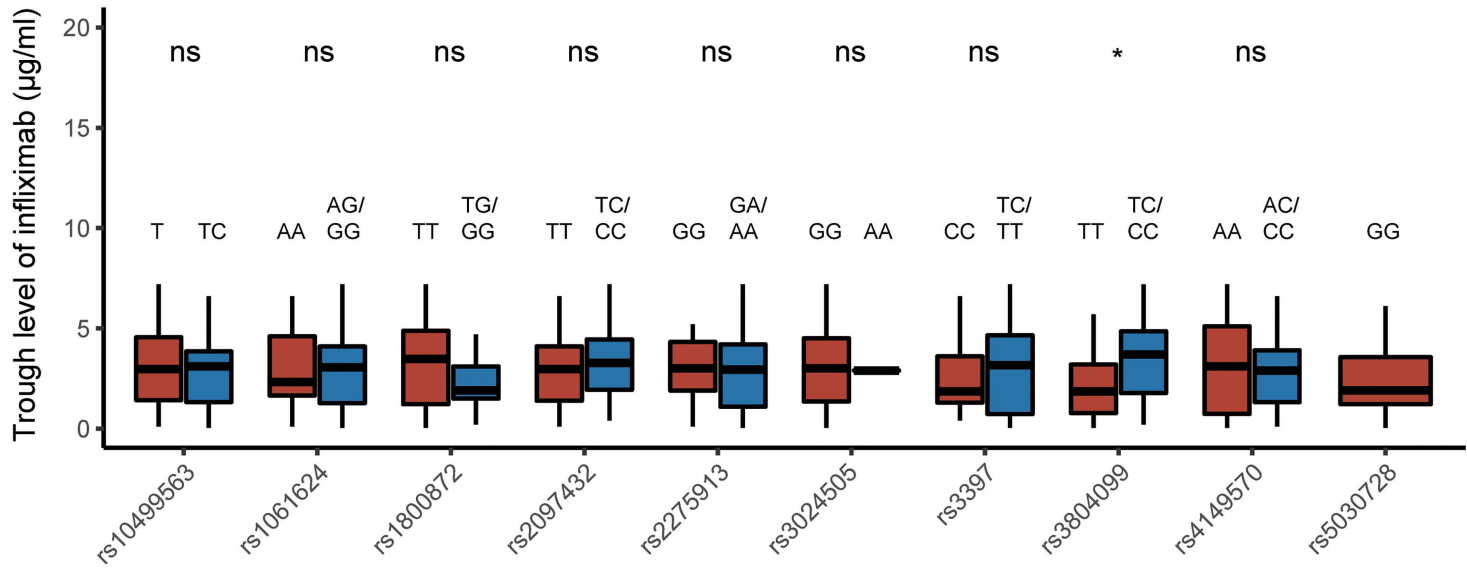

D

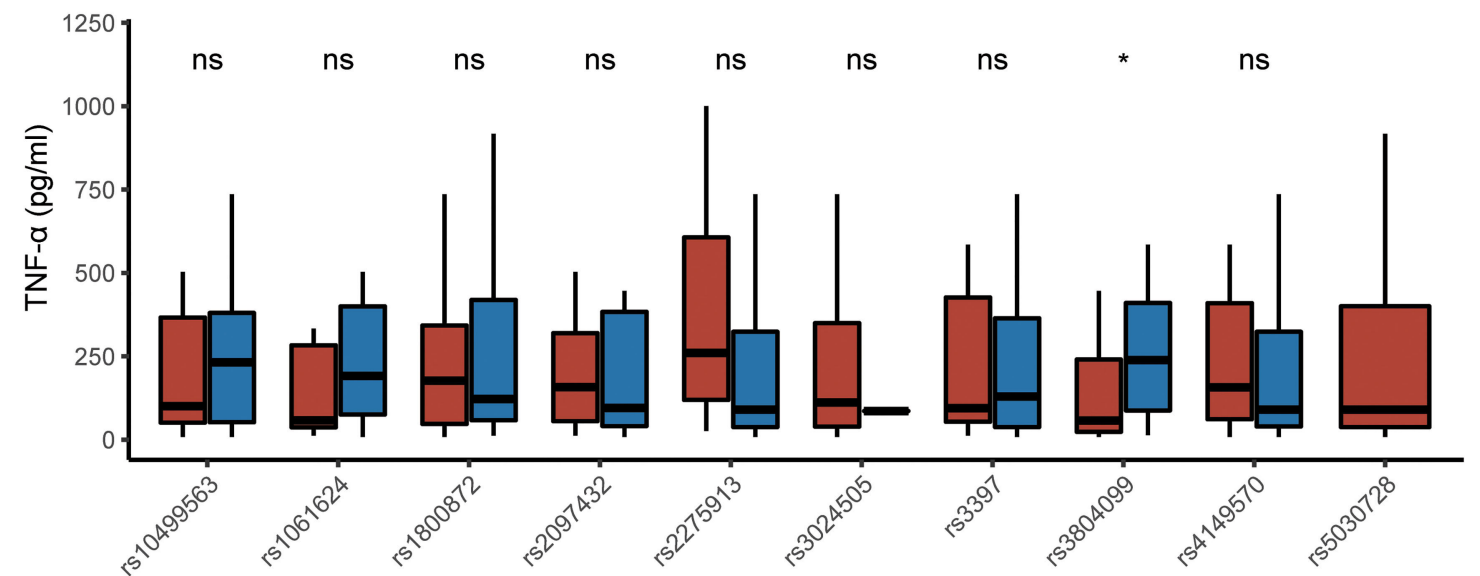

FIGURE 3 | SNPs associated with pharmacokinetics of IFX in CD. (A) Kaplan-Meier curve depicting the difference in antibody-free probability between patients with the CC genotype and those with the TC/TT genotype. (B) Forest plot showing the results of multivariate Cox regression model. (C) Polymorphisms associated with TLI at week 14. (D) Polymorphisms associated with serum TNF- $\alpha$ level at week 14. SNPs, single-nucleotide polymorphisms; IFX, infliximab; CD, Crohn's disease; TNF- $\alpha$, tumor necrosis factor alpha. 
week 14, SES-CD score before IFX treatment, and concomitant immunosuppressive therapy, TNFRSF1B CC genotype remained a significant risk factor for the generation of ATI (Figure 3B).

Moreover, compared with patients carrying the rs3804099 TC and CC genotype, those carrying the rs3804099 TT genotype had a lower median TLI (3.7 vs. $2.1 \mu \mathrm{g} / \mathrm{ml}, p=0.039)$ and serum TNF- $\alpha$ level (239.0 vs. $71.8 \mathrm{pg} / \mathrm{ml}, p=0.034)$ at week 14. The remaining SNPs were not statistically significant in the comparison of TLI and TNF- $\alpha$ level at week 14 (Figures 3C,D).

\section{DISCUSSION}

In the present study, 62 patients with $\mathrm{CD}$ treated with IFX were evaluated, and factors associated with $\mathrm{CR}$ and $\mathrm{MH}$ were identified. Notably, we identified one SNP (rs3397) as an independent risk factor for the presence of serum ATI. To our knowledge, this is the first study to investigate the role of pharmacogenetics in ATI development among pediatric patients with CD in China.

The association between TLI and clinical or endoscopic outcomes was observed at weeks 14 and 30. Singh et al. found that TLI and CRP at week 14 were significantly associated with persistent efficacy within 1 year (26). Ungar et al. found that TLI $>2.2 \mu \mathrm{g} / \mathrm{ml}$ at week 6 could predict sustained response for 1 year of IFX treatment (7). However, a relationship between TLI and long-term clinical response was not observed in this study, which was possibly due to the dose adjustment in some patients during the maintenance treatment and the small sample size.

Some studies showed that higher TLIs were associated with better clinical outcomes (27). A prospective observational study revealed that the optimal cutoff for TLI associated with CR and CRP normalization was $2.1 \mu \mathrm{g} / \mathrm{ml}$, while that for TLI associated with CR with normal CRP and fecal calprotectin $(<50 \mu \mathrm{g} / \mathrm{g})$ was $4.9 \mu \mathrm{g} / \mathrm{ml}$ in adults with inflammatory bowel disease (IBD) (28). Another observational study reported that TLI $>3.11 \mu \mathrm{g} / \mathrm{ml}$ at week 14 predicted sustained CR (week 54) in pediatric IBD (29). For predicting $\mathrm{MH}$, Chaparro et al. demonstrated that the best cutoff point of TLI was $3.4 \mu \mathrm{g} / \mathrm{ml}$ with an AUROC of 0.63 in adult patients (30). In this study, the optimal cutoff value of TLI for identifying patients in $\mathrm{MH}(3.34 \mu \mathrm{g} / \mathrm{ml})$ was consistent with some previously reported data for adults (28, 30). IFX pharmacokinetic properties were comparable between pediatric and adult CD patients (31). On the other hand, the cutoff levels in our study were also lower than some other studies. Kang et al. considered that the cutoff value of TLI for achieving $\mathrm{MH}$ was $\geq 5 \mu \mathrm{g} / \mathrm{ml}$ with a specificity of $80 \%$ in children (32). However, the definition of $\mathrm{MH}$ in that study was an SES-CD of 0 point. A more stringent expected level of clinical outcome was associated with a higher optimal threshold (28). Another retrospective study proposed that a therapeutic level of $6-10 \mu \mathrm{g} / \mathrm{ml}$ for IFX was required for $\mathrm{MH}$, but the trough levels of blood samples were not detected (33). Moreover, the individual variations in pharmacokinetics of IFX, such as gender, ethnicity, body mass index, development of immunogenicity, and disease severity, influenced the IFX pharmacokinetics (34).
Significantly, there might be substantial discrepancies between different commercial enzyme-linked immunosorbent assay kits for monitoring TLI (35). Therefore, it is recommended to use the same assay during follow-up and cautiously compare results issued by different kits.

Furthermore, we found that the ATI detection rate was quite low at week 14, which might have been due to the early testing time point. As the treatment progressed, the number of patients with positive ATI levels increased, and the median time to antibody detection was 13.48 months. Our result is consistent with a retrospective study that revealed a time to antibody detection for IFX of 14.83 months (36). In contrast to one previous study, which demonstrated that ATI in the early treatment course could predict long-term outcomes (37), no association between ATI and clinical outcomes was observed in the present study. Nevertheless, TLI was inversely correlated with ATI levels during the maintenance treatment, and factors affecting ATI occurrence were assessed.

During the evaluation of SNPs, one SNP (rs3397) in the TNFRSF1B gene was found to be significantly associated with time to ATI production. Interestingly, rs3397 was previously found to be associated with long-term response to anti-TNF treatment in pediatric CD patients (13). TNFRSF1B (TNF receptor superfamily $1 \mathrm{~B}$ ) is a single transmembrane glycoprotein that can induce cell survival or cell apoptosis (38). A recent study demonstrated that the $\mathrm{C}$ allele of $\mathrm{rs} 3397$ was associated with subtherapeutic trough serum levels of adalimumab $(<5 \mu \mathrm{g} / \mathrm{ml})$ (12). No data about this SNP have been reported in terms of ATI production. Although rs3397 was not found to be related to TLI in our study, the multivariate Cox regression analysis confirmed that the C allele for SNP rs3397 in TNFRSF1B was associated with a higher risk of earlier ATI production. The poorer outcome for patients diagnosed with $\mathrm{CD}$ with the CC genotype in rs3397 than in those with CT or TT suggests that genotyping this SNP could help to identify patients who are prone to immunogenicity to drugs. Bank et al. (23) reported that the TC or CC genotype of rs3804099, another polymorphism in TLR2 regulating the activation of the NF- $\kappa \mathrm{B}$ pathway, was associated with a beneficial response among $\mathrm{CD}$ patients. However, our observation demonstrated that patients carrying the rs3804099 TC and CC genotype expressed higher TLI and serum TNF- $\alpha$ levels at week 14 . Since an early increase in soluble TNF- $\alpha$ during IFX therapy might reflect a better long-term clinical outcome (39), our finding provides more evidence for the potential clinical application of rs3804099. Notably, rs5030728 in TLR4 was identified to be associated with subtherapeutic TLI in Spanish pediatric IBD (12), but all patients detected for rs5030728 were G allele carriers in our cohort, which might indicate a difference in pharmacogenetics among various populations. More studies are required to further confirm the role of this SNP as a biomarker for IFX treatment in pediatric CD.

The limitations of this study were its retrospective design, the relatively small number of enrolled patients, and missing data. Considering that the incidence of pediatric IBD is lower in China than that in Western countries (40) and that this center is one of the largest pediatric IBD centers in China, the enrolled population is considered representative. Moreover, a longer 
follow-up time may have resulted in a higher ATI detection rate compared with this present study. Despite these limitations, the identification of the role of TLI in clinical efficacy and SNP in ATI development provides more evidence that TDM or genotyping may optimize IFX treatment in pediatric CD patients in China.

In conclusion, this study indicates an association between TLI and short-term treatment outcomes in pediatric CD patients. The identification of the cutoff value for TLI contributing to $\mathrm{MH}$ in pediatric $\mathrm{CD}$ patients may guide adjustments of drug dosing in the treat-to-target approach. The C allele for SNP rs3397 in TNFRSF1B was found to be associated with a higher risk of earlier ATI production.

\section{DATA AVAILABILITY STATEMENT}

The original contributions presented in the study are included in the article/Supplementary Materials, further inquiries can be directed to the corresponding author/s.

\section{ETHICS STATEMENT}

This study was approved by the ethical committee at the Children's Hospital of Fudan University. Informed consents for participation and samples collection were obtained from their parents.

\section{REFERENCES}

1. Aloi M, Nuti F, Stronati L, Cucchiara S. Advances in the medical management of paediatric IBD. Nat Rev Gastroenterol Hepatol. (2014) 11:99108. doi: $10.1038 /$ nrgastro. 2013.158

2. Sands BE, Anderson FH, Bernstein CN, Chey WY, Feagan BG, Fedorak $\mathrm{RN}$, et al. Infliximab maintenance therapy for fistulizing Crohn's disease. $N$ Engl J Med. (2004) 350:876-85. doi: 10.1056/NEJMoa0 30815

3. Tajiri H, Motoya S, Kinjo F, Maemoto A, Matsumoto T, Sato N, et al. Infliximab for pediatric patients with Crohn's disease: a Phase 3, openlabel, uncontrolled, multicenter trial in Japan. PLoS ONE. (2018) 13:e201956. doi: 10.1371/journal.pone.0201956

4. Ding NS, Hart A, De Cruz P. Systematic review: predicting and optimising response to anti-TNF therapy in Crohn's disease - algorithm for practical management. Aliment Pharmacol Ther. (2016) 43:30-51. doi: 10.1111/apt.13445

5. D'Arcangelo G, Oliva S, Dilillo A, Viola F, Civitelli F, Isoldi S, et al. Predictors of long-term clinical and endoscopic remission in children with crohn disease treated with infliximab. J Pediatr Gastroenterol Nutr. (2019) 68:841-6. doi: 10.1097/MPG.0000000000002262

6. Berends SE, D'Haens G, Schaap T, de Vries A, Rispens T, Bloem $\mathrm{K}$, et al. Dried blood samples can support monitoring of infliximab concentrations in patients with inflammatory bowel disease: a clinical validation. $\mathrm{Br}$ J Clin Pharmacol. (2019) 85:1544-51. doi: 10.1111/bcp. 13939

7. Ungar B, Glidai Y, Yavzori M, Picard O, Fudim E, Lahad A, et al. Association between infliximab drug and antibody levels and therapy outcome in pediatric inflammatory bowel diseases. J Pediatr Gastroenterol Nutr. (2018) 67:507-12. doi: 10.1097/MPG.0000000000002051

8. Paul S, Del TE, Marotte H, Rinaudo-Gaujous M, Moreau A, Phelip JM, et al. Therapeutic drug monitoring of infliximab and mucosal healing in inflammatory bowel disease: a prospective study. Inflamm Bowel Dis. (2013) 19:2568-76. doi: 10.1097/MIB.0b013e3182a77b41

\section{AUTHOR CONTRIBUTIONS}

WH performed the experiments, analyzed the data, and drafted the manuscript. $\mathrm{ZY}$ and $\mathrm{YH}$ revised the manuscript. YF, ZT, YW, and LQ were responsible for the associated data collection. All authors contributed to the article and approved the submitted version.

\section{FUNDING}

This study was supported by the National Children's Medical Center, Haiju International Joint Lab Fund (EK1125180105).

\section{ACKNOWLEDGMENTS}

The authors would like to thank all patients and families participating in the study.

\section{SUPPLEMENTARY MATERIAL}

The Supplementary Material for this article can be found online at: https://www.frontiersin.org/articles/10.3389/fped. 2021.744599/full\#supplementary-material

Supplementary Figure S1 | Illustration of infliximab therapeutic protocol for pediatric CD patients at our center. IFX, infliximab; EEN, exclusive enteral nutrition; TDM, Therapeutic drug monitoring.

9. Minar P, Saeed SA, Afreen M, Kim MO, Denson LA. Practical use of infliximab concentration monitoring in pediatric crohn disease. J Pediatr Gastroenterol Nutr. (2016) 62:715-22. doi: 10.1097/MPG.00000000000 01029

10. Vande CN, Herfarth $\mathrm{H}$, Katz J, Falck-Ytter Y, Singh S. American gastroenterological association institute technical review on the role of therapeutic drug monitoring in the management of inflammatory bowel diseases. Gastroenterology. (2017) 153:835-57. doi: 10.1053/j.gastro.2017.07.031

11. Sazonovs A, Kennedy NA, Moutsianas L, Heap GA, Rice DL, Reppell M, et al. HLA-DQA ${ }^{*} 05$ carriage associated with development of anti-drug antibodies to infliximab and adalimumab in patients with crohn's disease. Gastroenterology. (2020) 158:189-99. doi: 10.1053/j.gastro.2019.09.041

12. Salvador-Martín S, Pujol-Muncunill G, Bossacoma F, Navas-López VM, Gallego-Fernández C, Segarra O, et al. Pharmacogenetics of trough serum anti-TNF levels in paediatric inflammatory bowel disease. Br J Clin Pharmacol. (2021) 87:447-57. doi: 10.1111/bcp.14400

13. Salvador-Martín S, Bossacoma F, Pujol-Muncunill G, Navas-López VM, Gallego-Fernández C, Viada J, et al. Genetic predictors of long-term response to antitumor necrosis factor agents in pediatric inflammatory bowel disease. J Pediatr Gastroenterol Nutr. (2020) 71:508-15. doi: 10.1097/MPG.0000000000002840

14. Levine A, Koletzko S, Turner D, Escher JC, Cucchiara S, de Ridder L, et al. ESPGHAN revised porto criteria for the diagnosis of inflammatory bowel disease in children and adolescents. J Pediatr Gastroenterol Nutr. (2014) 58:795-806. doi: 10.1097/MPG.0000000000000239

15. Levine A, Griffiths A, Markowitz J, Wilson DC, Turner D, Russell RK, et al. Pediatric modification of the montreal classification for inflammatory bowel disease: the Paris classification. Inflamm Bowel Dis. (2011) 17:1314-21. doi: 10.1002/ibd.21493

16. Swaminath A, Feathers A, Ananthakrishnan AN, Falzon L, Li FS. Systematic review with meta-analysis: enteral nutrition therapy for the induction of remission in paediatric Crohn's disease. Aliment Pharmacol Ther. (2017) 46:645-56. doi: 10.1111/apt.14253 
17. Hansen T, Duerksen DR. Enteral nutrition in the management of pediatric and adult crohn's disease. Nutrients. (2018) 10:537. doi: 10.3390/nu10050537

18. Mack DR, Benchimol EI, Critch J, DeBruyn J, Tse F, Moayyedi P, et al. Canadian association of gastroenterology clinical practice guideline for the medical management of pediatric luminal crohn's disease. Gastroenterology. (2019) 157:320-48. doi: 10.1053/j.gastro.2019.03.022

19. Turner D, Griffiths AM, Walters TD, Seah T, Markowitz J, Pfefferkorn M, et al. Mathematical weighting of the pediatric Crohn's disease activity index (PCDAI) and comparison with its other short versions. Inflamm Bowel Dis. (2012) 18:55-62. doi: 10.1002/ibd.21649

20. Schaffer T, Schoepfer AM, Seibold F. Serum ficolin-2 correlates worse than fecal calprotectin and CRP with endoscopic Crohn's disease activity. J Crohns Colitis. (2014) 8:1125-32. doi: 10.1016/j.crohns.2014.02.014

21. van Rheenen PF, Aloi M, Assa A, Bronsky J, Escher JC, Fagerberg UL, et al. The medical management of paediatric crohn's disease: an ECCO-ESPGHAN guideline update. J Crohns Colitis. (2020). doi: 10.1093/ecco-jcc/jjaa161. [Epub ahead of print].

22. Matsukura $\mathrm{H}$, Ikeda S, Yoshimura $\mathrm{N}$, Takazoe $\mathrm{M}$, Muramatsu $\mathrm{M}$. Genetic polymorphisms of tumour necrosis factor receptor superfamily $1 \mathrm{~A}$ and $1 \mathrm{~B}$ affect responses to infliximab in Japanese patients with Crohn's disease. Aliment Pharmacol Ther. (2008) 27:765-70. doi: 10.1111/j.1365-2036.2008.03630.x

23. Bank S, Andersen PS, Burisch J, Pedersen N, Roug S, Galsgaard J, et al. Associations between functional polymorphisms in the NFкB signaling pathway and response to anti-TNF treatment in Danish patients with inflammatory bowel disease. Pharmacogenomics J. (2014) 14:526-34. doi: $10.1038 /$ tpj.2014.19

24. Salvador-Martín S, López-Cauce B, Nuñez O, Laserna-Mendieta EJ, García MI, Lobato E, et al. Genetic predictors of long-term response and trough levels of infliximab in crohn's disease. Pharmacol Res. (2019) 149:104478. doi: 10.1016/j.phrs.2019.104478

25. Frank EHJ. Rms: Regression Modeling Strategies. Available online at: http:// CRAN.R-project.org/package=rms (accessed March 18, 2021).

26. Singh N, Rosenthal CJ, Melmed GY, Mirocha J, Farrior S, Callejas S, et al. Early infliximab trough levels are associated with persistent remission in pediatric patients with inflammatory bowel disease. Inflamm Bowel Dis. (2014) 20:1708-13. doi: 10.1097/MIB.0000000000000137

27. Franca R, Curci D, Lucafo M, Decorti G, Stocco G. Therapeutic drug monitoring to improve outcome of anti-TNF drugs in pediatric inflammatory bowel disease. Expert Opin Drug Metab Toxicol. (2019) 15:527-39. doi: 10.1080/17425255.2019.1630378

28. Roblin X, Boschetti G, Duru G, Williet N, Deltedesco E, Phelip JM, et al. Distinct thresholds of infliximab trough level are associated with different therapeutic outcomes in patients with inflammatory bowel disease. Inflamm Bowel Dis. (2017) 23:2048-53. doi: 10.1097/MIB.0000000000 01223

29. Naviglio S, Lacorte D, Lucafò M, Cifù A, Favretto D, Cuzzoni E, et al. Causes of treatment failure in children with inflammatory bowel disease treated with infliximab. J Pediatr Gastr Nutr. (2019) 68:37-44. doi: 10.1097/MPG.0000000000002112

30. Chaparro M, Barreiro-de Acosta M, Echarri A, Almendros R, Barrio J, Llao J, et al. Correlation between anti-TNF serum levels and endoscopic inflammation in inflammatory bowel disease patients. Digest Dis Sci. (2019) 64:846-54. doi: 10.1007/s10620-018-5362-3

31. Fasanmade AA, Adedokun OJ, Blank M, Zhou H, Davis HM. Pharmacokinetic properties of infliximab in children and adults with Crohn's disease: a retrospective analysis of data from 2 phase III clinical trials. Clin Ther. (2011) 33:946-64. doi: 10.1016/j.clinthera.2011.06.002

32. Kang B, Choi SY, Choi YO, Lee SY, Baek SY, Sohn I, et al. Infliximab trough levels are associated with mucosal healing during maintenance treatment with infliximab in paediatric crohn's disease. J Crohns Colitis. (2019) 13:189-97. doi: 10.1093/ecco-jcc/jjy155

33. Ungar B, Levy I, Yavne Y, Yavzori M, Picard O, Fudim E, et al. Optimizing antiTNF- $\alpha$ therapy: serum levels of infliximab and adalimumab are associated with mucosal healing in patients with inflammatory bowel diseases. Clin Gastroenterol Hepatol. (2016) 14:550-7. doi: 10.1016/j.cgh.2015.10.025

34. Ordás I, Feagan BG, Sandborn WJ. Therapeutic drug monitoring of tumor necrosis factor antagonists in inflammatory bowel disease. Clin Gastroenterol H. (2012) 10:1079-87. doi: 10.1016/j.cgh.2012.06.032

35. Bertin D, Serrero M, Grimaud JC, Desjeux A, Desplat-Jégo S. Monitoring of infliximab trough levels and anti-infliximab antibodies in inflammatory bowel diseases: a comparison of three commercially available ELISA kits. Cytokine. (2020) 126:154859. doi: 10.1016/j.cyto.2019.154859

36. Moses J, Lambert-Jenkins K, Momotaz H, Sattar A, Debanne SM, Splawski $J$, et al. Time to antibody detection and associated factors for presence of anti-drug antibodies in pediatric inflammatory bowel disease patients treated with anti-TNF therapy. Eur J Gastroen Hepat. (2019) 31:1228-33. doi: 10.1097/MEG.0000000000001538

37. Stein R, Lee D, Leonard MB, Thayu M, Denson LA, Chuang E, et al. Serum infliximab, antidrug antibodies, and tumor necrosis factor predict sustained response in pediatric crohn's disease. Inflamm Bowel Dis. (2016) 22:1370-7. doi: 10.1097/MIB.0000000000000769

38. Papadakis KA, Targan SR. Tumor necrosis factor: biology and therapeutic inhibitors. Gastroenterology. (2000) 119:1148-57. doi: 10.1053 /gast.2000.18160

39. Eder P, Korybalska K, Łykowska-Szuber L, Stawczyk-Eder K, KrelaKazmierczak I, Łuczak J, et al. An increase in serum tumour necrosis factor- $\alpha$ during anti-tumour necrosis factor- $\alpha$ therapy for Crohn's disease - a paradox or a predictive index? Dig Liver Dis. (2016) 48:1168-71. doi: 10.1016/j.dld.2016.06.038

40. Sýkora J, Pomahačová R, Kreslová M, Cvalínová D, Štych P, Schwarz J. Current global trends in the incidence of pediatric-onset inflammatory bowel disease. World J Gastroenterol. (2018) 24:2741-63. doi: 10.3748/wjg.v24.i25.2741

Conflict of Interest: The authors declare that the research was conducted in the absence of any commercial or financial relationships that could be construed as a potential conflict of interest.

Publisher's Note: All claims expressed in this article are solely those of the authors and do not necessarily represent those of their affiliated organizations, or those of the publisher, the editors and the reviewers. Any product that may be evaluated in this article, or claim that may be made by its manufacturer, is not guaranteed or endorsed by the publisher.

Copyright (C) 2021 Hu, Feng, Ye, Tang, Qian, Wang and Huang. This is an openaccess article distributed under the terms of the Creative Commons Attribution License (CC BY). The use, distribution or reproduction in other forums is permitted provided the original author(s) and the copyright owner(s) are credited and that the original publication in this journal is cited, in accordance with accepted academic practice. No use, distribution or reproduction is permitted which does not comply with these terms. 